\title{
Charm Physics at BESIII
}

\section{Pei-Rong Li*, Yu Zhang}

University of Chinese Academy of Sciences

E-mail: Lipeirong11dmails.ucas.ac.cn

The BESIII Experiment at the Beijing Electron Positron Collider (BEPCII) has accumulated the world's largest $e^{+} e^{-}$collision samples at $\psi(3770)$ peak, around the $\psi(4040)$ nominal mass, and at the $\Lambda_{c}^{+} \bar{\Lambda}_{c}^{-}$pair mass threshold which allow us to study decays of charmed mesons and baryons in a uniquely clean background. We report the measurements of the $D_{(s)}^{+}$decay constants, the form factors of $D$ semi-leptonic decays, the CKM matrix elements $\left|V_{c s(d)}\right|$, the $D_{(s)}^{+}$hadronic decay branching fractions, and the absolute branching fractions of the hadronic and semi-leptonic decays of $\Lambda_{c}^{+}$.

16th International Conference on B-Physics at Frontier Machines 2-6 May, 2016

Marseille, France

${ }^{*}$ Speaker. 


\section{Introduction}

In the Standard Model (SM), the Cabibbo-Kobayashi-Maskawa (CKM) matrix parametrizes the mixing between the quark flavors in the weak interaction. The CKM matrix elements $\left|V_{c s(d)}\right|$ could be well determined via precision measurements of the form factors in semi-leptonic $D$ decays, as they are parameterized by the decay constants $f_{D}$ and the CKM matrix elements $\left|V_{c s(d)}\right|$. With these measurements, we are able to calibrate the LQCD calculations and test the CKM matrix unitary.

Moreover, current theoretical calculations of the charmed mesons partial decay widths are not consistent well with experimental results as the $c$ quark mass is not heavy enough for good convergence of the heavy quark expansion nor light enough for chiral perturbative theory. Yet, hadronic decays of charmed mesons provide important information on flavor mixing, $C P$ violation, and strong-interaction effects [四. Precision measurements of the hadronic decay rates also strengthen the understanding of $U$-spin and $S U(3)$-flavor symmetry breaking effects in $D$ decays [ [వ].

Furthermore, unlike the charmed meson decays, charmed baryon decays suffer from great uncertainties experimentally. Thus, the improved measurement of the charmed baryon $\Lambda_{c}^{+}$decays is of crucial importance for that they could both provide plentiful information on the dynamics in the charm sector and improved input for beauty physics.

In this paper, the recent results of the semi-leptonic and hadronic decays of $D^{0}, D_{(s)}^{+}$and $\Lambda_{c}^{+}$ are reported. These studies are based on 2.93, 0.482 and $0.567 \mathrm{fb}^{-1}$ data at $\sqrt{s}=3.773,4.009$ and $4.599 \mathrm{GeV}$ collected with the BESIII Experiment, where $D^{0} \bar{D}^{0}, D^{+} D^{-}, D_{s}^{+} D_{s}^{-}$and $\Lambda_{c}^{+} \bar{\Lambda}_{c}^{-}$are produced in pairs.

\section{2. $D$ semi-leptonic decays}

Charmed mesons can decay into lighter hadrons by emitting a $l^{+} v_{l}$ pair via a virtual $W^{+}$ boson. These semi-leptonic decays can be parameterized by the quark mixing matrix element and the form factor of hadronic weak current. In the limit of zero positron mass, the differential rate for $D^{0} \rightarrow K^{-}\left(\pi^{-}\right) e^{+} v_{e}$ decay is given by

$$
\frac{d \Gamma}{d q^{2}}=\frac{G_{F}^{2}}{24 \pi^{3}}\left|V_{c s(d)}\right|^{2}\left|p_{K(\pi)}\right|^{3}\left|f_{+}^{K(\pi)}\left(q^{2}\right)\right|^{2},
$$

where $G_{F}$ is the Fermi coupling constant, $\left|V_{c s(d)}\right|$ is the quark mixing element, $p_{K(\pi)}$ is the threemomentum of the kaon(pion) in the rest frame of the $D^{0}$, and $f_{+}^{K(\pi)}\left(q^{2}\right)$ is the hadronic form factor of the hadronic weak current depending on the square of the four-momentum transfer $q=$ $p_{D^{0}}-p_{K(\pi)}$.

Generally, three forms of parameterizations are used to model the form factors as follows:

- the simple model $f_{+}\left(q^{2}\right)=\frac{f_{+}(0)}{1-\frac{q^{2}}{M_{\text {pole }}}}$, where the pole mass $M_{\text {pole }}$ is a free parameter;

- the modified model $f_{+}\left(q^{2}\right)=\frac{f_{+}(0)}{\left(1-\frac{q^{2}}{M_{\text {pole }}^{2}}\right)\left(1-\alpha \frac{q^{2}}{M_{\text {pole }}^{2}}\right)}$, where only $\alpha$ is a free parameter and $M_{\text {pole }}$ is fixed in this case; 
- the series expansion $f_{+}(t)=\frac{1}{P(t) \Phi\left(t, t_{0}\right)} a_{0}\left(t_{0}\right)\left(1+\sum_{k=1}^{\infty} r_{k}\left(t_{0}\right)\left[z\left(t, t_{0}\right)\right]^{k}\right)$ [四].

Using a double tag method, the singly tagged $\bar{D}^{0}$ meson is reconstructed using five hadronic decays: $K^{+} \pi^{-}, K^{+} \pi^{-} \pi^{0}, K^{+} \pi^{-} \pi^{+} \pi^{-}, K^{+} \pi^{-} \pi^{0} \pi^{0}, K^{+} \pi^{-} \pi^{+} \pi^{-} \pi^{0}$, as shown in Fig. Ш $(\mathrm{g}$ k). Based on the observed 70727.0 \pm 278.3 signals for $D^{0} \rightarrow K^{-} e^{+} v_{e}$ and $6297.1 \pm 86.8$ signals for $D^{0} \rightarrow \pi^{-} e^{+} v_{e}$ [可], their branching fractions are determined to be $\mathscr{B}\left(D^{0} \rightarrow K^{-} e^{+} v_{e}\right)=$ $\left(3.505 \pm 0.014_{\text {stat. }} \pm 0.033_{\text {sys. }}\right) \%$ and $\mathscr{B}\left(D^{0} \rightarrow \pi^{-} e^{+} v_{e}\right)=\left(0.295 \pm 0.004_{\text {stat. }} \pm 0.003_{\text {sys. }}\right) \%$ respectively. Fig. $\square$ shows the fits to partial decay widths using the simple pole model, modified pole model and the series expansion. Using the values for $f_{+}^{K}(\pi)(0)\left|V_{c s(d)}\right|$ from the two-parameter $z$-series expansion fits and with $f_{+}^{K}(0)=0.747 \pm 0.011 \pm 0.015$ [可] and $f_{+}^{\pi}(0)=0.666 \pm 0.020 \pm 0.021$ [四] calculated in LQCD, we obtain $\left|V_{c s}\right|=0.9601 \pm 0.0033 \pm 0.0047 \pm 0.0239$ and $\left|V_{c d}\right|=0.2155 \pm$ $0.0027 \pm 0.0014 \pm 0.0094$, where the first uncertainties are statistical, the second ones systematic, and the third ones are due to the theoretical uncertainties in the form factor calculations. From the measured ratio of $\frac{f_{+}^{\pi}(0)\left|V_{c d}\right|}{f_{+}^{K}(0)\left|V_{c s}\right|}$ together with the LCSR calculation of $f_{+}^{\pi}(0) / f_{+}^{K}(0)=0.84 \pm 0.04$ [ []], the ratio $\frac{\left|V_{c c}\right|}{\left|V_{c s}\right|}$ is obtained to be $0.238 \pm 0.004 \pm 0.002 \pm 0.011$, where the first error is statistical, the second one systematic, and the third one is from LCSR normalization. BESIII results are in good agreement with the previous measurements, and have the best precision up to date.

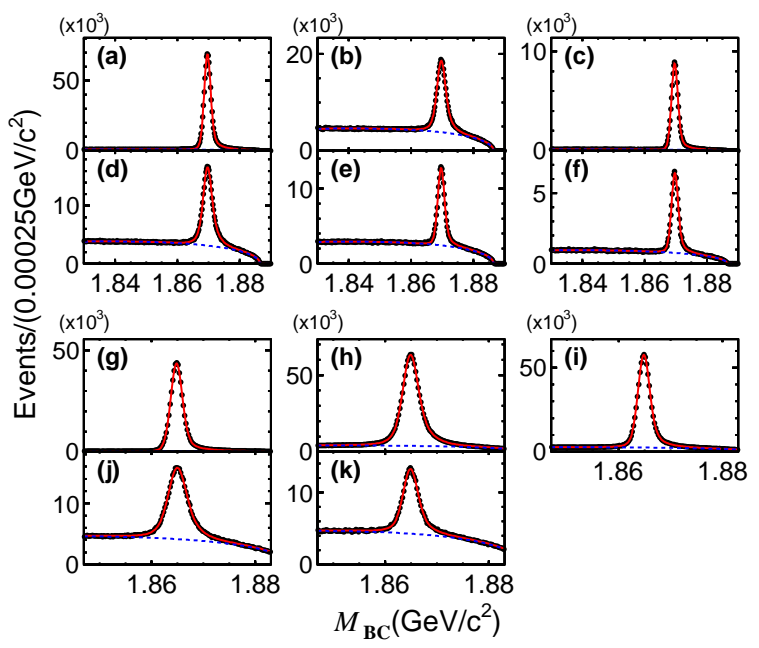

Figure 1: $M_{B C}$ distributions of single tag samples for different tag modes. The first two rows show charged $D$ decays: (a) $K^{+} \pi^{-} \pi^{-}$, (b) $K^{+} \pi^{-} \pi^{-} \pi^{0}$, (c) $K_{s} \pi^{-}$, (d) $K_{s} \pi^{-} \pi^{0}$, (e) $K_{s} \pi^{+} \pi^{-} \pi^{-}$, (f) $K^{+} K^{-} \pi^{-}$, the latter two rows show neutral $D$ decays: (g) $K^{+} \pi^{-}$, (h) $K^{+} \pi^{-} \pi^{0}$, (i) $K^{+} \pi^{-} \pi^{+} \pi^{-}$, (j) $K^{+} \pi^{-} \pi^{0} \pi^{0}$, (k) $K^{+} \pi^{-} \pi^{+} \pi^{-} \pi^{0}$. Data are shown as points, the (red) solid lines are the total fits and the (blue) dashed lines are the background shapes. $D$ and $\bar{D}$ candidates are combined.

Similarly, using the six single tag modes $K^{+} \pi^{-} \pi^{-}, K^{+} \pi^{-} \pi^{-} \pi^{0}, K_{S} \pi^{-}, K_{s} \pi^{-} \pi^{0}, K_{S} \pi^{+} \pi^{-} \pi^{-}$, $K^{+} K^{-} \pi^{-}$as shown in Fig. $\mathbf{W}(\mathrm{a}-\mathrm{f})$, the semi-leptonic decays $D^{+} \rightarrow(\omega / \phi) e^{+} v_{e}$ and $D^{0} \rightarrow K_{L}^{0} e^{+} v_{e}$ are studied. The first measurement of the absolute branching fraction $\mathscr{B}\left(D^{0} \rightarrow K_{L}^{0} e^{+} v_{e}\right)$ is determined to be $\left(4.481 \pm 0.027_{\text {stat. }} \pm 0.103_{\text {sys. }}\right) \%$ [ [ ] ], which agrees well with previous measurement of $\mathscr{B}\left(D^{+} \rightarrow K_{s} e^{+} v_{e}\right)$. The $C P$ asymmetry $A_{C P}^{D^{0} \rightarrow K_{L}^{0} e^{+} v_{e}}=\left(-0.59 \pm 0.60_{\text {stat. }} \pm 1.48_{\text {sys. }}\right) \%$, which indicates no $\mathrm{CP}$ asymmetry exists in this decay, is also determined. Furthermore, a simultaneous 


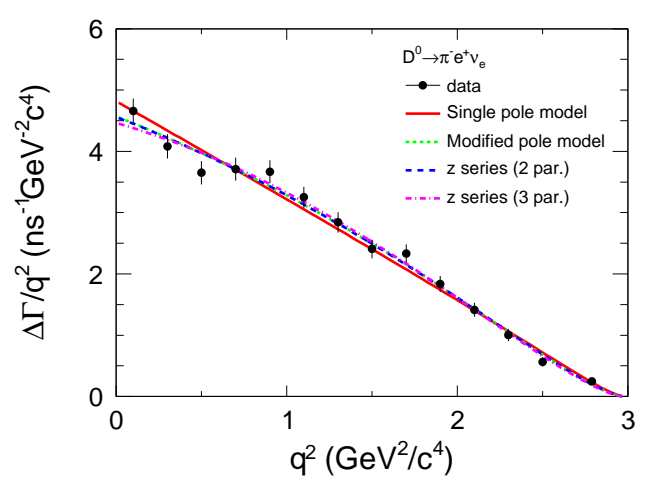

(a)

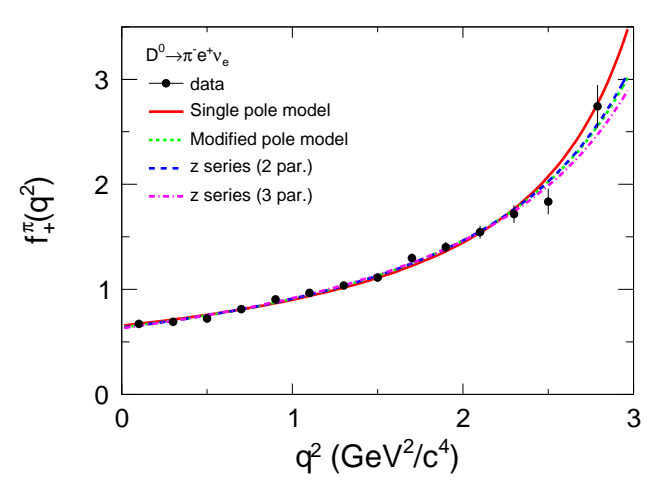

(b)

Figure 2: Fits to partial decay widths of (a) $D^{0} \rightarrow K^{-} e^{+} v_{e}$ and (b) $D^{0} \rightarrow \pi^{-} e^{+} v_{e}$

fit is performed to the event density $I\left(q^{2}\right)$ for the six tag modes with the series expansion model, and the product of $f_{K}^{+}(0)\left|V_{c S}\right|$ is obtained to be $0.728 \pm 0.006_{\text {stat. }} \pm 0.011_{\text {sys. }}$.

The yield of the decay $D^{+} \rightarrow \omega e^{+} v_{e}$ obtained using the six tag modes is $491 \pm 32[$ [ $]$. With these signals, the absolute branching fraction for the decay is measured to be $\left(1.63 \pm 0.11_{\text {stat. }} \pm\right.$ $\left.0.08_{\text {sys. }}\right) \times 10^{-3}$, which represents a significant improvement compared to previous measurements. Besides, the ratio of hadronic form factors is also obtained to be $r_{V}=\frac{V(0)}{A_{1}(0)}=1.24 \pm 0.09_{\text {stat }} . \pm$ $0.06_{\text {sys. }}$ and $r_{2}=\frac{A_{2}(0)}{A_{1}(0)}=1.06 \pm 0.15_{\text {stat. }} \pm 0.05_{\text {sys. }}$. No significant signal is observed in the search for $D^{+} \rightarrow \phi e^{+} v_{e}$, and hence an upper limit at the $90 \%$ confidence level (C.L.) is set to be $\mathscr{B}\left(D^{+} \rightarrow\right.$ $\left.\phi e^{+} v_{e}\right)<1.3 \times 10^{-5}$.

\section{3. $D$ hadronic decays}

The singly Cabibbo-suppressed (SCS) decays $D^{+, 0} \rightarrow \omega \pi^{+, 0}$ have not yet been observed, and only upper limits at $90 \%$ C.L. on the branching fractions are set to be $3.4 \times 10^{-4}$ and $2.6 \times 10^{-4}$, respectively, by the CLEO Collaboration[ए]]. Theoretically, the $D \rightarrow \omega \pi$ decays should be at about $1.0 \times 10^{-4}$ level []] . Based on the double tag method, six tag modes for charged $D$ and

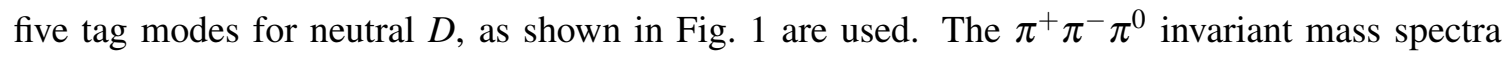
are fitted as shown in Fig [ 1 . This is the first observation of the SCS decay $D^{+} \rightarrow \omega \pi^{+}$with statistical significance of $5.5 \sigma$, and the first evidence for the SCS decay $D^{0} \rightarrow \omega \pi^{0}$ with statistical significance of $4.1 \sigma$. The results are consistent with the theoretical prediction [س]].

Recently, an updated measurement of $\mathscr{B}\left(D_{s}^{+} \rightarrow \eta^{\prime} \pi^{+} \pi^{0}\right)=(5.6 \pm 0.5 \pm 0.6) \%$ [ए2] is reported by CLEO, which is much smaller than the previous result [13]]. Based on the single tag events, where $D_{s}^{-}$is reconstructed via nine tag modes: $K_{S}^{0} K^{-}, K^{+} K^{-} \pi^{-}, K^{+} K^{-} \pi^{-} \pi^{0}, K_{S}^{0} K^{+} \pi^{-} \pi^{-}$, $\pi^{+} \pi^{-} \pi^{-}, \pi^{-} \eta, \pi^{-} \eta^{\prime}\left(\eta^{\prime} \rightarrow \pi^{+} \pi^{-} \eta\right), \pi^{-} \eta^{\prime}\left(\eta^{\prime} \rightarrow \rho^{0} \gamma, \rho^{0} \rightarrow \pi^{+} \pi^{-}\right)$, and $\pi^{-} \pi^{0} \eta$, the branching fraction $\mathscr{B}\left(D_{s}^{+} \rightarrow \eta^{\prime} X\right)=(8.8 \pm 1.8 \pm 0.5) \%$ is found, which is consistent with previous measurement [U4]]. $\mathscr{B}\left(D_{s}^{+} \rightarrow \eta^{\prime} \rho^{+}\right)$is obtained to be $(5.8 \pm 1.4 \pm 0.4) \%$, which is compatible with CLEO's updated measurement [[2], in which the resonant process $\eta^{\prime} \rho^{+}$is believed to dominate. 


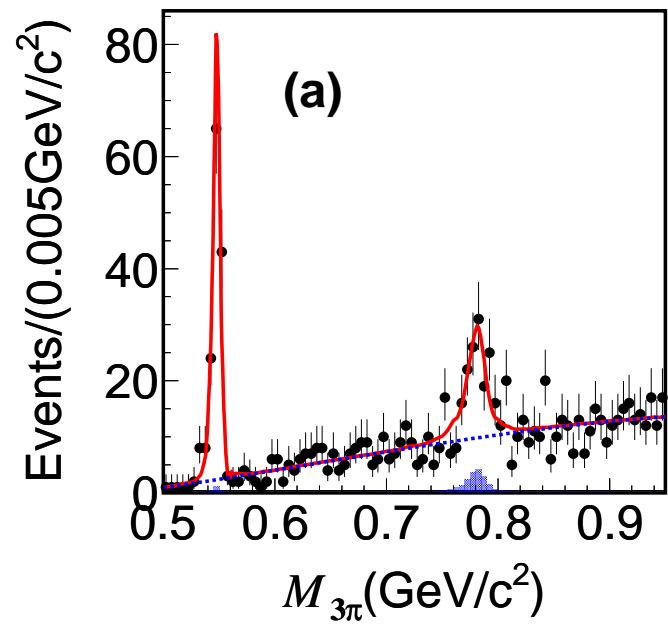

(a)

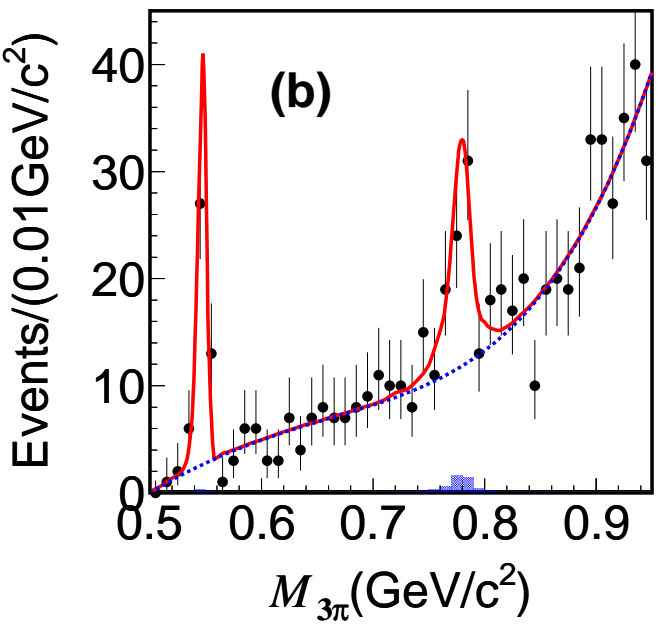

(b)

Figure 3: Fits to the $3 \pi$ mass spectra for (a) $D^{+} \rightarrow \pi^{+} \pi^{-} \pi^{0} \pi^{+}$and (b) $D^{0} \rightarrow \pi^{+} \pi^{-} \pi^{0} \pi^{0}$. The (red) solid lines are the total fits, the (blue) dashed lines are the background shapes, and the hatched histograms are peaking background

\section{4. $\Lambda_{c}^{+}$decays}

A data sample at $\sqrt{s}=4.599 \mathrm{GeV}$ collected by BESIII allows to perform the first absolute measurement of $\Lambda_{c}^{+}$decays. Twelve Cabibbo-favored $\Lambda_{c}^{+}$decay rates are measured by employing a double tag technique [15]. About 15 thousand of the singly tagged $\Lambda_{c}^{+}$events are observed,

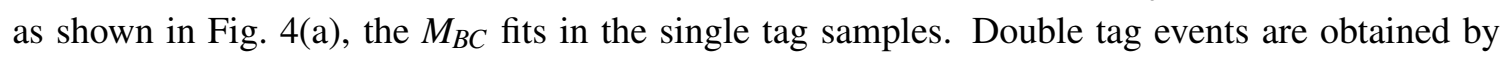
fitting to the $M_{B C}$ distributions as shown in Fig. $Q(\mathrm{~b})$. The branching fractions of the twelve modes are determined to be $\mathscr{B}\left(p K_{S}^{0}\right)=(1.52 \pm 0.08 \pm 0.03) \%, \mathscr{B}\left(p K^{-} \pi^{+}\right)=(5.84 \pm 0.27 \pm 0.23) \%$, $\mathscr{B}\left(p K_{S}^{0} \pi^{0}\right)=(1.87 \pm 0.13 \pm 0.05) \%, \mathscr{B}\left(p K_{S}^{0} \pi^{+} \pi^{-}\right)=(1.53 \pm 0.11 \pm 0.09) \%, \mathscr{B}\left(p K^{-} \pi^{+} \pi^{0}\right)=$ $(4.53 \pm 0.23 \pm 0.30) \%, \mathscr{B}\left(\Lambda \pi^{+}\right)=(1.24 \pm 0.07 \pm 0.03) \%, \mathscr{B}\left(\Lambda \pi^{+} \pi^{0}\right)=(7.01 \pm 0.37 \pm 0.19) \%$, $\mathscr{B}\left(\Lambda \pi^{+} \pi^{-} \pi^{+}\right)=(3.81 \pm 0.24 \pm 0.18) \%, \mathscr{B}\left(\Sigma^{0} \pi^{+}\right)=(1.27 \pm 0.08 \pm 0.03) \%, \mathscr{B}\left(\Sigma^{+} \pi^{0}\right)=(1.18 \pm$ $0.10 \pm 0.03) \%, \mathscr{B}\left(\Sigma^{+} \pi^{+} \pi^{-}\right)=(4.25 \pm 0.24 \pm 0.20) \%, \mathscr{B}\left(\Sigma^{+} \omega\right)=(1.56 \pm 0.20 \pm 0.07) \%$. These results improve the PDG values precision [16].

The first absolute measurement of the $\Lambda_{c}^{+}$semi-leptonic decay $\Lambda_{c}^{+} \rightarrow \Lambda e^{+} v_{e}$ is also performed using the twelve tag modes except $\Sigma^{+} \omega$ as shown in Fig. $ه($ a). $103.5 \pm 10.9$ signals are observed, and the branching fraction is $\mathscr{B}\left(\Lambda_{c}^{+} \rightarrow \Lambda e^{+} v_{e}\right)=\left(3.63 \pm 0.38_{\text {stat. }} \pm 0.20_{\text {sys. }}\right) \%$. The result can serve as a valuable test of non-perturbtive models and LQCD calculations.

\section{5. summary}

To summarize, by analysing 2.93, 0.482 and $0.567 \mathrm{fb}^{-1}$ data taken at $\sqrt{s}=3.773,4.009$ and $4.599 \mathrm{GeV}$ with the BESIII detector, the decay constants, the form factors of $D$ semi-leptonic decays are reported, which provide important test to LQCD calculations, and enable tests of CKM matrix unitary. The study of charmed meson hadronic decays is given with significantly improved precision. Also, the first absolute measurements of charmed baron decays are performed. All these 


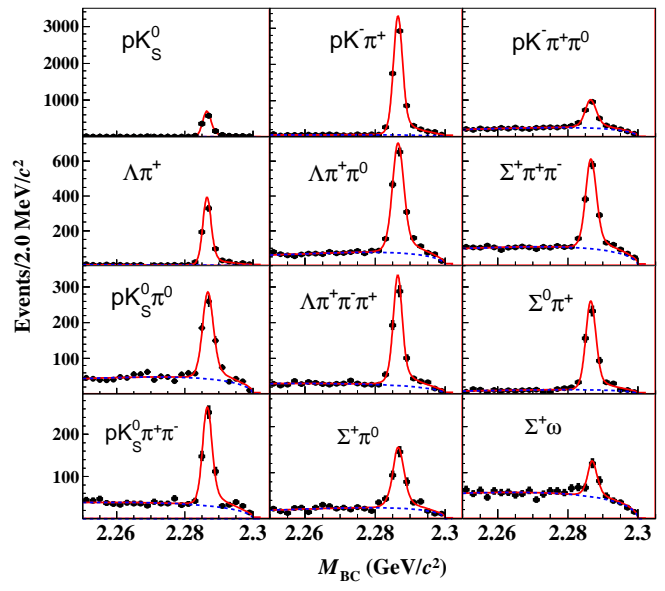

(a)

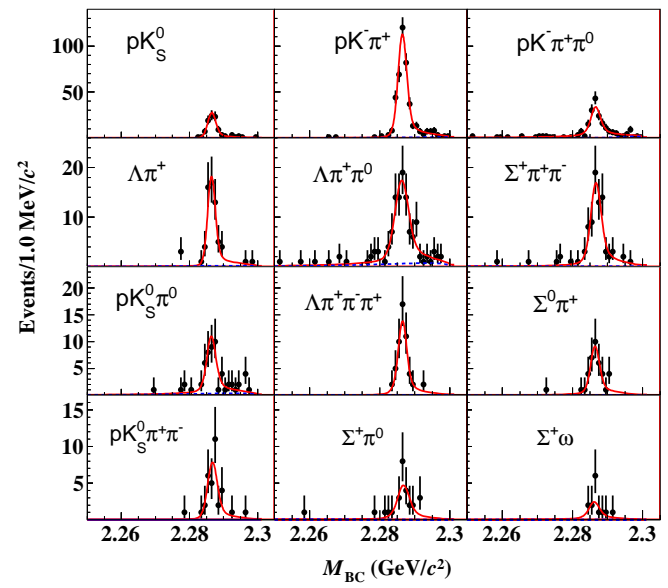

(b)

Figure 4: (a) Fits to the single tag $M_{B C}$ distributions in data for the different decay modes. (b) Fits to the double tag $M_{B C}$ distributions in data for different signal modes. Points with error bars are data, solid lines are the sum of the fit functions, and dashed lines are the background shapes.

could improve understanding of non-perturbative QCD. It is worthwhile to mention that BESIII will take $D_{s} D_{s}^{*}$ data at $4.180 \mathrm{GeV}$, which would further help in understanding the $D_{s}$ physics.

\section{References}

[1] M. Artuso, B. Meadows and A. A. Petrov, Ann. Rev. Nucl. Part. Sci. 58, 249 (2008).

[2] W. Kwong and S. P. Rosen, Phys. Lett. B 298, 413 (1993); Y. Grossman and D. J. Robinson, JHEP 1304, 067 (2013).

[3] C.G. Boyd, B. Grinstein, and R.F. Lebed, Nucl. Phys. B 461, 493 (1996).

[4] M. Ablikim et al. (BESIII Collaboration), Phy. Rev. D 92, 072012 (2015).

[5] P. Ball, Phys. Lett. B 641, 50 (2006).

[6] H. Na et al. (HPQCD Collaboration), Phys. Rev. D 82, 114506 (2010).

[7] H. Na et al. (HPQCD Collaboration), Phys. Rev. D 84, 114505 (2011).

[8] M. Ablikim et al. (BESIII Collaboration), Phys.Rev. D 92, 112008 (2015).

[9] M. Ablikim et al. (BESIII Collaboration), Phys.Rev. D 92, 071101 (2015).

[10] P. Rubin et al. (CLEO Collaboration), Phys. Rev. Lett. 96, 081802 (2006).

[11] H. Y. Cheng and C. W. Chiang, Phys. Rev. D 81, 074021 (2010).

[12] P. U. E. Onyisi et al. (CLEO Collaboration), Phys. Rev. D 88, 032009 (2013).

[13] C. Jessop et al. (CLEO Collaboration), Phys. Rev. D 58, 052002 (1998).

[14] M. Ablikim et al. (BESIII Collaboration), Physics Letters B 750, 466 (2015).

[15] M. Ablikim et al. (BESIII Collaboration), Phys.Rev.Lett 116, 052001 (2016).

[16] K. A. Olive et al. (Particle Data Group), Chin. Phys. C 38,090001 (2014). 